\title{
Method for the evaluation of minor losses in pulsatile laminar fluid flow
}

\author{
H. Netřebská ${ }^{1, a}$, J. Matěcha ${ }^{1}$, M. Schmirler ${ }^{1}$, L. Manoch $^{1}$, J. Adamec $^{1}$ \\ ${ }^{1}$ CTU in Prague, Faculty of Mechanical Engineering, Department of Fluid Mechanics and Thermodynamics
}

\begin{abstract}
This article focuses on description and evaluation of the size of the minor losses in pulsatile laminar flow. It describes a method for determination of minor losses with respect to the phase delay between pressure waveforms and flow waveforms. The whole evaluation method is presented on the example of minor loss in the sharp $90^{\circ}$ bend in the channel with constant square cross-section in pulsatile laminar flow of an incompressible Newtonian fluid. For this example, the minor loss coefficient is expressed depending on the dimensionless flow parameters. Total minor loss was calculated numerically. An analytical solution was used for quantification of the head loss in the developed laminar flow in the straight channel. Unsteady flow conditions were controlled by the sinusoidal flow.
\end{abstract}

\section{Introduction}

In recent years, the attention has been concentrated on laminar pulsatile flow of fluids in non-round crosssection channels, mainly due to their increasing application in practice. These channels are used e.g. for mass or heat transfer in various solar systems [1], heat exchangers [2], blade machines, fuel cells $[3,4]$ etc. The issue of unsteady phenomena is also reflected in areas, which seem to be distant from engineering practice at first sight, such us blood flow in cardiovascular system $[5,6,7,8,9]$ or air flow in the respiratory system [10].

Currently, there is only few information about local losses in laminar flow and a lack of a comprehensive work, such as [11] for area of turbulent flow. It is possible to find values of coefficients of local losses for selected cases, e.g. in publications [12, 13]. In current works the values of local loss coefficients are determined for a specific application, especially from numerical simulation, which brings complications in the experimental research where the laminar flow is connected with measurement of small pressure differences and small flow rate. The aim of the paper is to describe local loss as coefficient that can be further used for two-dimensional flow models (curvilinear coordinate, time). In order to verify the method described above, a square cross-section channel with a sharp $90^{\circ}$ bend was chosen.

\section{Methods}

In order to evaluate local losses in the sharp bend of the square cross-section channel with dimensions $a$, the sinusoidal course of the pulsatile flow was divided to steady and oscillating component. The resulting loss is constituted by the sum of the local loss of steady flow and a friction loss of steady flow, local loss of oscillating flow, friction loss and influence of inertia forces of the oscillating flow. The issue of local loss is not linear and the local loss thus includes all factors influencing the flow, e.g. flow development downstream the local loss.

The loss for steady and oscillating component of flow in channel with a sharp bend is expressed by impedance $\hat{Z}$, which is a complex number, as a ratio of pressure difference in complex shape $\Delta \hat{P}$ and flow rate in complex shape $\hat{Q}$.

For steady component of the flow, the impedance has $\hat{Z}$ only real part

$$
Z_{s t}=\frac{\Delta p_{s t}}{q_{s t}}=Z_{\lambda s t}+Z_{\xi s t},
$$

which can be divided into impedance caused by friction losses $Z_{\lambda s t}$ and impedance of flow caused by local losses $Z_{\xi s t}$. For steady components of the pulsatile flow, the quantities $\Delta p_{s t}$ and $q_{s t}$ are the time average values of pressure and flow.

Local loss impedance of the steady component of the pulsatile flow $Z_{\xi_{s t}}$ is calculated as a difference between total calculated impedance $Z_{s t}$ of channel with a sharp bend and analytically calculated impedance of corresponding length of a straight section of channel of the same flow regime corresponding to friction losses $Z_{\lambda s t}[14]$ :

$$
Z_{\xi s t}=Z_{s t}-Z_{\lambda s t}
$$


$Z_{\lambda s t}$ is calculated according to [14] as:

$$
Z_{\lambda s t}=Z_{A N-s t}^{\|}=\frac{\Delta p_{A N-s t}^{\|}}{q_{s t}},
$$

where

$$
\Delta p_{A N-s t}^{\|}=q_{s t} \frac{3 \eta L}{4 a^{4}} \frac{1}{\left[1-\left(\frac{192}{\pi^{5}} \sum_{n=1,3,5 \ldots}^{\infty} \frac{\tanh \left(\frac{i \pi}{2}\right)}{n^{5}}\right)\right]},
$$

where $\eta$ is a dynamic viscosity and $\mathrm{L}$ is total channel length.

The total impedance of the oscillating component of the pulsatile flow is composed of the sum of the local loss impedance $\hat{Z}_{\xi o s c}$ and inertia forces impedance and friction losses impedance $\hat{Z}_{\text {wosc }}$.

$$
\hat{Z}_{o s c}=\frac{\Delta \hat{P}_{o s c} e^{i \omega t}}{\hat{Q}_{o s c} e^{i \omega t}}=\hat{Z}_{\xi o s c}+\hat{Z}_{\omega o s c}
$$

where $\Delta \hat{P}_{o s c} e^{i \omega t}$ is pressure expressed by complex number and likewise $\Delta \hat{Q}_{o s c} e^{i \omega t}$ is flow expressed by complex number and angular velocity $\omega=2 \pi f$, where $f$ is pulsatile flow frequency.

Impedance of inertia forces of the oscillating component of flow $\hat{Z}_{\text {wosc }}$ is calculated from the analytical solution as impedance of direct channel of corresponding length. For evaluation of local loss impedance $\hat{Z}_{\text {גosc }}$ it is necessary to deduct the impedance of friction losses and influence of inertia forces $\hat{Z}_{\text {wosc }}$ from the whole calculated impedance.

$$
\hat{Z}_{\xi o s c}=\hat{Z}_{o s c}-\hat{Z}_{\omega o s c} \text {. }
$$

$\hat{Z}_{\text {wosc }}$ is calculated according to [15] as:

$$
\begin{gathered}
\hat{Z}_{\omega o s c}=\hat{Z}_{A N-s t}^{\|}=\frac{\left|\hat{P}_{A N-o s c}^{\|}\right| e^{i\left(\omega t+\varphi_{0}\right)} e^{(i \varphi)}}{|\hat{Q}| e^{i\left(\omega t+\varphi_{0}\right)}}, \\
\Delta \hat{P}_{A N-s t}^{\|}=\frac{q_{A m p} i \eta \alpha^{2} L}{4(a / 2)^{4}} \frac{1}{\left[1-2 \sum_{n=0}^{\infty} \frac{1}{p_{n}^{2}}\left(2 \frac{\tanh \left(r_{n}\right)}{r_{n}}\right)\right]},
\end{gathered}
$$

where $q_{\text {Amp }}$ is amplitude of the oscillating flow, $i$ is complex unit, $\alpha$ is nondimensional frequency parameter calculated as $\alpha=\sqrt{\frac{2 \pi f}{v}} \frac{a}{2}$, coefficients $p_{n}$ and $r_{n}$ are defined as $p_{n}=\frac{\pi}{2}(2 n+1), r_{n}=\sqrt{i n^{2}+p_{n}^{2}}$.
The local loss impedance by pulsatile flow can be therefore characterize by three values: by local loss impedance of the steady component of flow $Z_{\xi s t}$, by the magnitude of local loss impedance of oscillating component of flow $\left|\hat{Z}_{\zeta \text { osc }}\right|$ and by value of phase shift $\varphi$ between oscillating course of pressure and flow rate.

Total pressure loss can be expressed by impedance for pulsatile flow in channel with a sharp bend as follows:

$$
\begin{aligned}
& \Delta p_{p u l}=Z_{\lambda s t} \cdot Q_{s t}+Z_{\xi s t} \cdot Q_{s t}+ \\
& +\mathfrak{R}\left(\hat{Z}_{\omega o s c} \hat{Q}_{o s c} e^{i \omega t}\right)+\mathfrak{R}\left(\hat{Z}_{\xi o s c} \hat{Q}_{o s c} e^{i \omega t}\right) .
\end{aligned}
$$

\section{Numerical solution}

The channel with a sharp bend was solved numerically. For numerical simulation, a geometric model was created in the Design Modeler program, which is part of the Workbench ANSYS v13 platform. The geometric model was designed with a cross-section size of $(5 \times 5) \mathrm{mm}$ and total length of $1.5 \mathrm{~m}$. The mesh was created by structured six-wall rectangular cells with a total of approx. 6 milion. (figure 1)

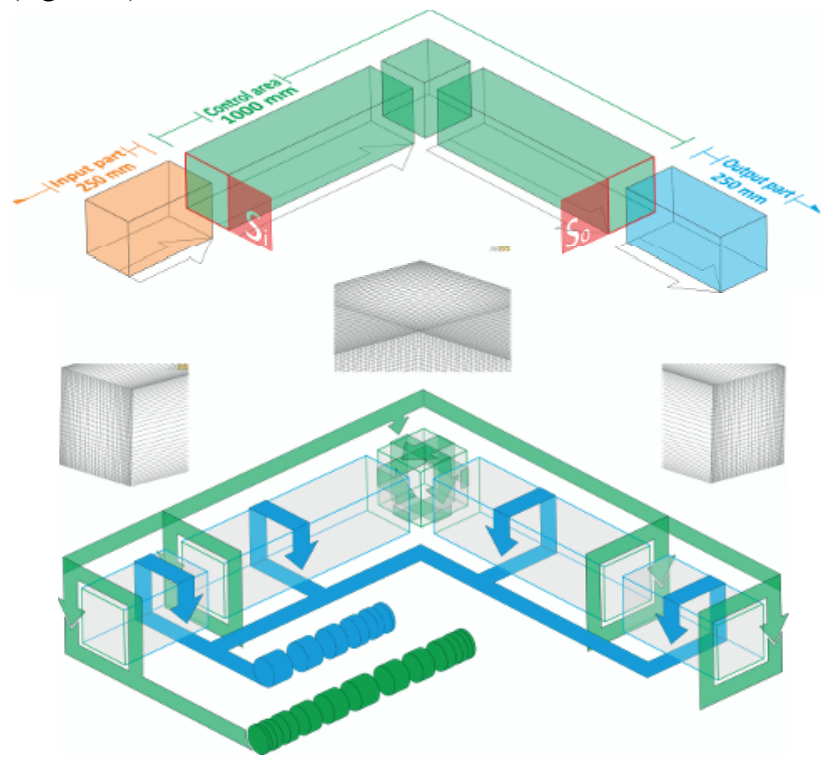

Fig. 1. Up - geometrical model of the channel, Middle details of the grid, Down - scheme of the cells division.

For the numerical simulation, the commercial solver ANSYS Fluent v13 was used. The flow was considered isothermal, unsteady, viscous, incompressible and laminar. A pressure based coupled solver was used in the simulation with the implicit scheme of second order of accuracy used for time and space discretization. The time step length was chosen T/720. At the model output, an atmospheric pressure in the whole border area was defined. At the model input, a rectangular velocity profile $v_{p u l}(t)=v_{s t}+v_{A m p} \sin (2 \pi f t)$. was prescribed with the help of udf function (user defined function). In the course of the solution, the values of the flow rate and static pressure in input and output area of the control volume were monitored and exported at every point in time and 
subsequently used for impedance calculation. Detailed information concerning the mesh of the model and setting of calculation is published in [16], where the numerical simulation results were compared to the results from the experiment, which was carried out with the help of Particle Image Velocimetry. The parameters of PIV measurement were chosen according to [17].

\section{Results}

The local loss impedance in the channel with a sharp bend was described by means of three values. The local loss impedance for steady component of flow, local loss impedance for oscillating component of flow and phase shift were described separately. For steady component of flow, the local loss impedance was described as $Z_{\zeta s t}$. For oscillating flow component, where the impedance is a complex number $\left|\hat{Z}_{\zeta \text { osc }}\right| e^{i \varphi}$, the impedance was evaluated separately as magnitude of impedance vector $\left|\hat{Z}_{\zeta \text { osc }}\right|$ and a phase shift $\varphi$.For the evaluation of influences of particular parameters of the pulsatile flow on the local loss, 13 flow regimes were calculated with particular regimes being described in table 1 . The pulsatile flow can be described by the values of Reynolds numbers $\operatorname{Re}(t)=\operatorname{Re}_{s t}+\operatorname{Re}_{A m p}(2 \pi f t)$ where $\operatorname{Re}_{s t}$ is value of Reynolds number for steady component of flow, $\operatorname{Re}_{A m p}$ is value of Reynolds number corresponding to the amplitude of the oscillating flow component. The frequency of the pulsatile flow is expressed by the frequency parameter $\alpha$. The local loss was evaluated with regard to the influence of steady component of flow $\operatorname{Re}_{s t}$ (regimes no. 1-5), the magnitude of the amplitude of the oscillating component of flow $\mathrm{Re}_{A m p}$ (regimes no.

3 , 6-9) and with regard to the frequency parameter $\alpha$ (regimes no. $3,10-13$ ).

Tab. 1. The list of computed regimes.

\begin{tabular}{|c|c|c|c|c|}
\hline $\begin{array}{l}\text { Regime } \\
\text { number }\end{array}$ & $\mathrm{Re}_{s t}$ & $\mathrm{Re}_{A m p}$ & $\alpha$ & $\zeta$ \\
\hline 1 & 400 & 300 & 3.95 & 0.01 \\
\hline 2 & 500 & 300 & 3.95 & 0.019 \\
\hline 3 & 600 & 300 & 3.95 & 0.013 \\
\hline 4 & 700 & 300 & 3.95 & 0.013 \\
\hline 5 & 800 & 300 & 3.95 & 0.034 \\
\hline 6 & 600 & 100 & 3.95 & 0.018 \\
\hline 7 & 600 & 200 & 3.95 & 0.013 \\
\hline 8 & 600 & 300 & 3.95 & 0.023 \\
\hline 9 & 600 & 400 & 3.95 & 0.038 \\
\hline 10 & 600 & 300 & 1.97 & 0.038 \\
\hline 11 & 600 & 300 & 2.8 & 0.048 \\
\hline 12 & 600 & 300 & 3.42 & 0.022 \\
\hline 13 & 600 & 300 & 4.42 & 0.014 \\
\hline
\end{tabular}

Influence of the steady component of the pulsatile flow on local loss

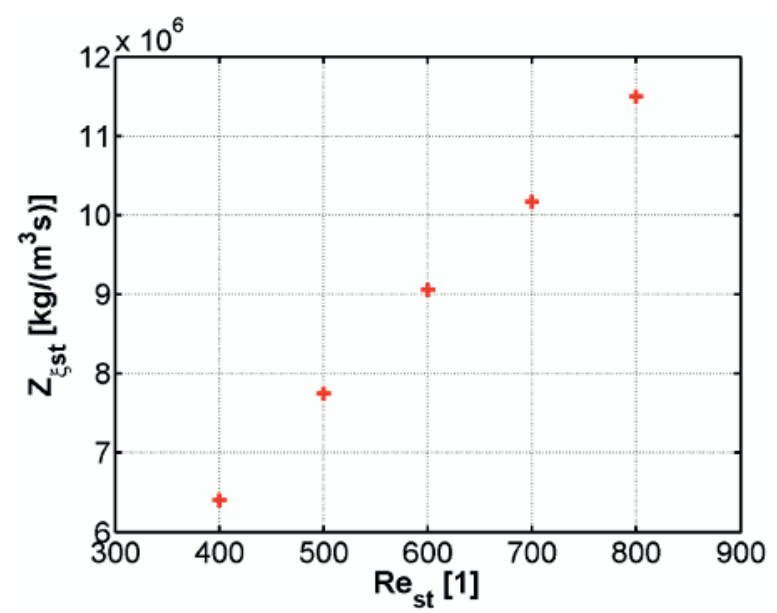

Fig. 2. Dependence of local loss impedance of steady component of pulsatile flow on steady component of pulsatile flow.

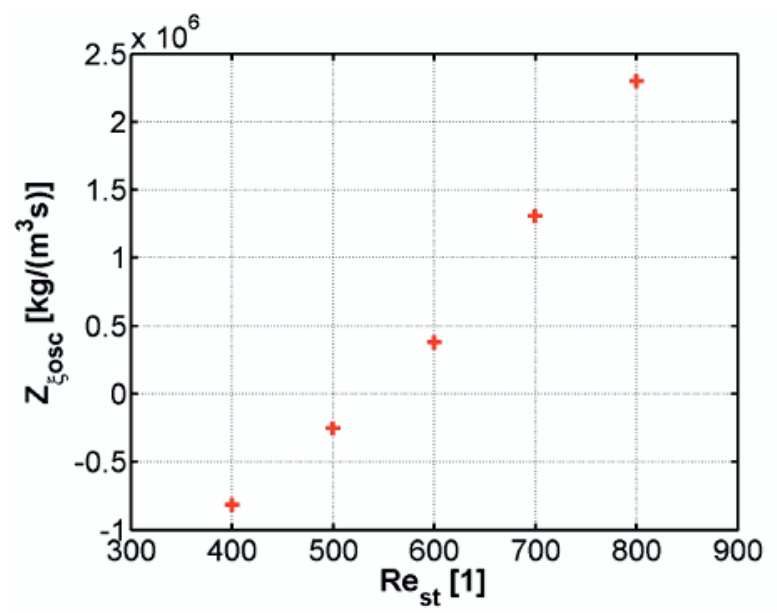

Fig. 3. Influence of local loss impedance of oscillating component of pulsatile flow in dependence on steady component of pulsatile flow.

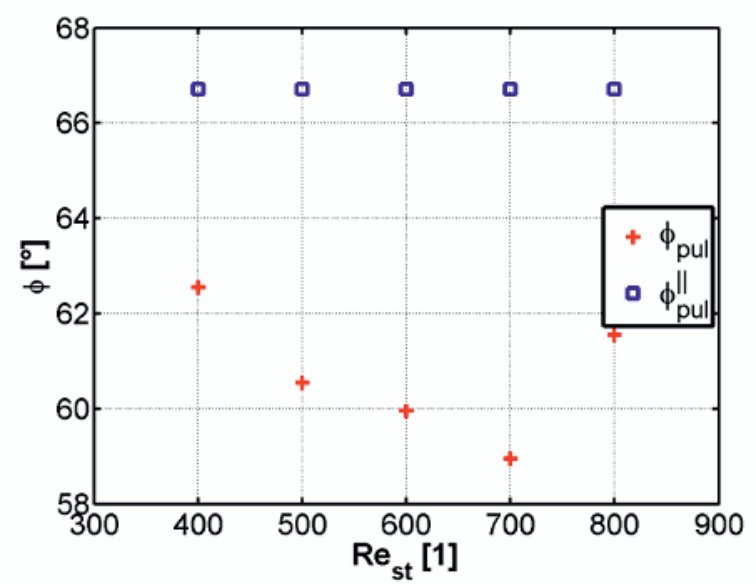

Fig. 4. Dependence of phase shift of pressure and flow in channel with bend in comparison with analytical solution of straight channel for various steady components of flow. 
Influence of amplitude of the oscillating component of flow on local loss

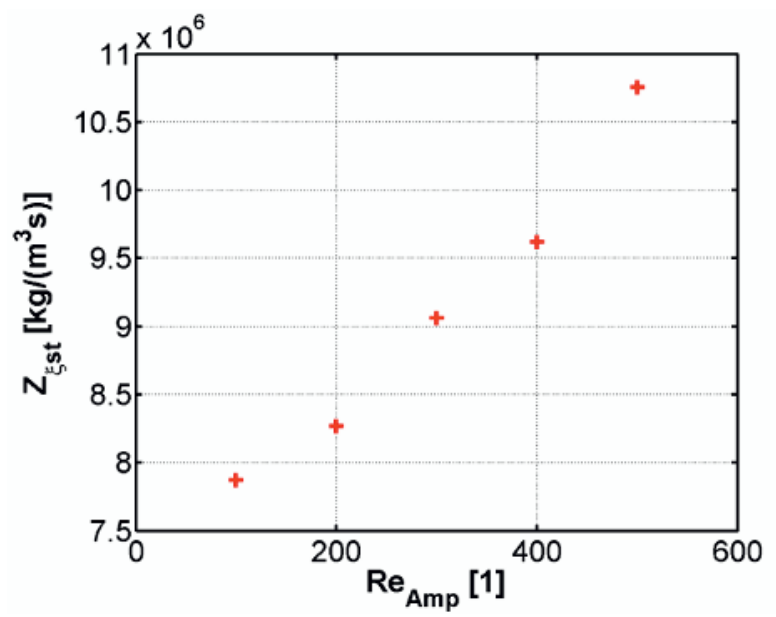

Fig. 5. Dependence of local loss impedance of steady component of pulsatile flow on amplitude magnitude of oscillating component of pulsatile flow.

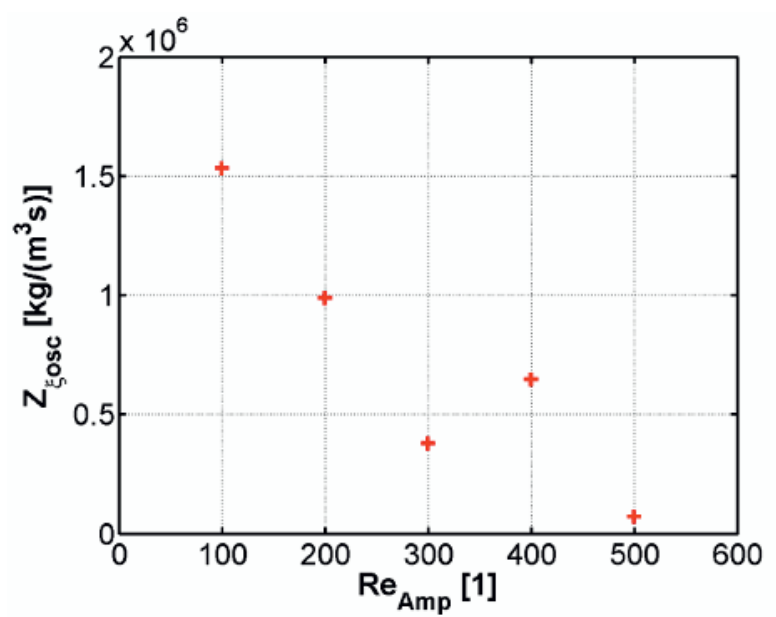

Fig. 6. Dependence of local loss impedance of oscillating component of pulsatile flow on amplitude magnitude of oscillating component of pulsatile flow.

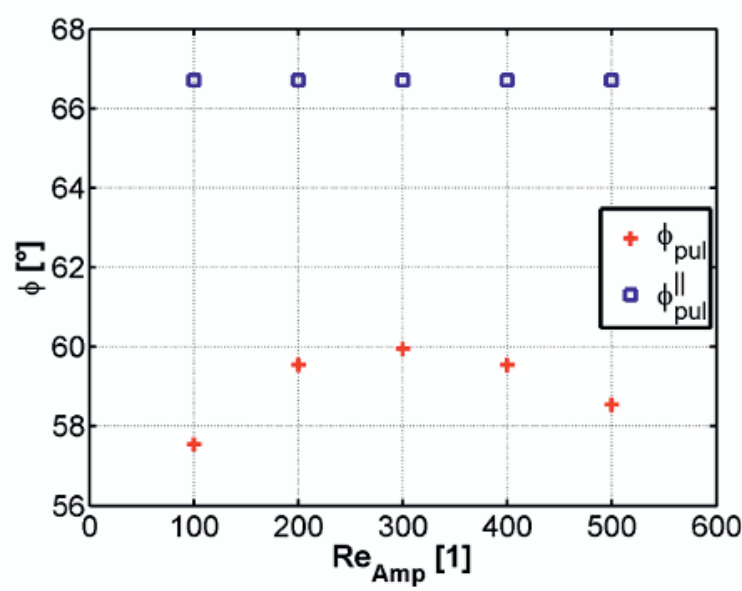

Fig. 7. Dependence of phase shift of pressure and the flow in channel with bend in comparison with analytical solution of straight channel for various steady components of flow.
Influence of pulsatile flow frekvency on local loss

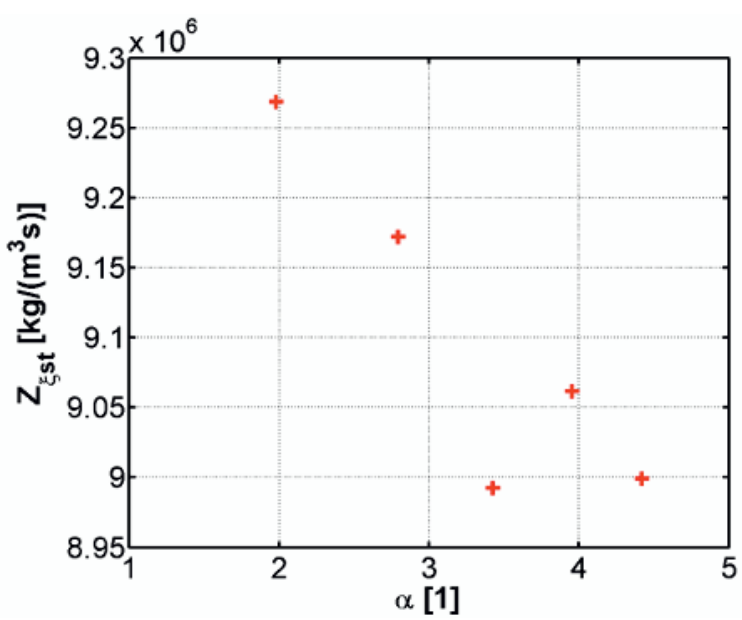

Fig. 8. Dependence of local loss impedance of steady component of pulsatile flow on frequency parameter of flow.

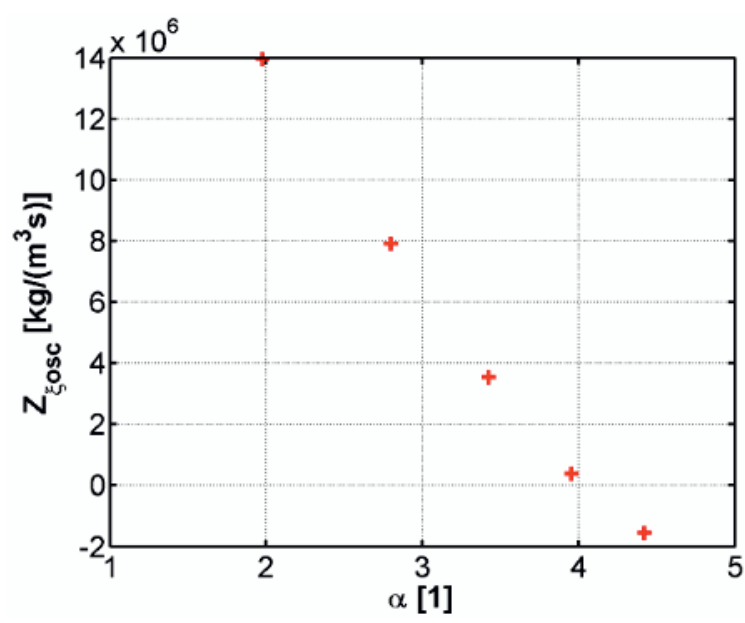

Fig. 9. Dependence of local loss impedance of oscillating component of pulsatile flow on frequency parameter of flow.

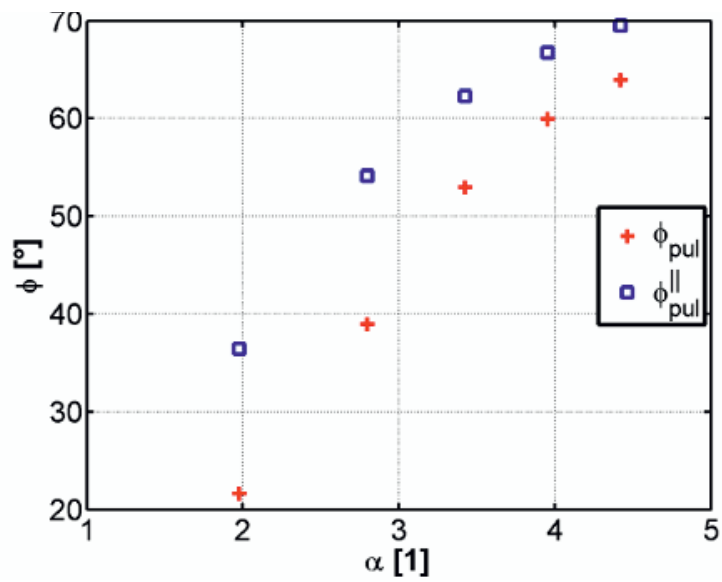

Fig. 10. Dependence of phase shift of pressure and the flow in channel with bend in comparison with analytical solution of straight channel for various frequency parameters of flow. 


\section{Conclusion}

The paper introduces a procedure for evaluation of local loss magnitude in pulsatile flow and for quantification of the local loss by means of established impedance, which consists of real number for steady component of flow and of complex number for oscillating component of flow. The whole procedure was verified on local loss of the sharp bend of the square channel. For verification of the procedure the total pressure loss of the channel $\Delta p_{p u l}$ was retroactively calculated from the calculated results of local loss impedance according to equation (6). This pressure loss was compared, for control, with pressure loss recorded during numerical simulation $\Delta p_{\text {pul-Num }}$ (figure 11,12). The comparison was used for evaluation of the error $\zeta=\left(\Delta p_{p u l}-\Delta p_{p u l-N u m}\right) /\left(\Delta p_{p u l-N u m}\right)_{\max }$ that is listed for particular regimes in Tab. 1. The largest error $4.8 \%$ was recorded for flow regime no. 11 .

The influences of particular flow parameters on local loss described by impedance were evaluated from the presented results (table 2). When the steady component of the pulsatile flow is increasing, the value of $Z_{\zeta s t}$ and $\left|\hat{Z}_{\xi o s c}\right|$ increases as well, the phase shift magnitude $\varphi$ is decreasing (figure 2-4). With the increasing oscillating component of the pulsatile flow $\operatorname{Re}_{\text {Amp }}$ the local loss impedance of steady flow $Z_{\xi s t}$ increases against the local loss impedance of oscillating component $\left|\hat{Z}_{\xi o s c}\right|$ decreases and the phase shift does not indicate a significant trend (figure 5-7). With increasing frequency parameter $\alpha$ both local loss impedances $Z_{\xi_{s t}}$ as well as $\left|\hat{Z}_{\xi o s c}\right|$ decrease and the phase shift $\varphi$ decreases (figure 8-10).

Table 2. Influences of parameters of pulsatile flow on values of local loss impedance, arrows indicate decreasing or increasing dependencies between particular quantities.

\begin{tabular}{|c||c|c|c|}
\hline & $\operatorname{Re}_{s t}$ & $\operatorname{Re}_{A m p}$ & $\alpha$ \\
\hline \hline$Z_{\xi s t}$ & \multicolumn{1}{|c|}{} & \multicolumn{1}{|c|}{} \\
\hline$\left|\hat{Z}_{\xi o s c}\right|$ & \multicolumn{1}{|c|}{} & & \\
\hline$\varphi$ & $\square$ & $\square$ & \\
\hline
\end{tabular}

\section{Acknowledgements}

This research has been supported by MSM6840770035 The Development of Environmental - Friendly Decentralized Power Engineering (2007-2013, MSM) and EU Regional Development Fund in OP R\&D for Innovations (OP VaVpI) and Ministry for Education, Czech Republic, project \# CZ.1.05/2.1.00/03.0125 Acquisition of Technology for Vehicle Center of Sustainable Mobility.

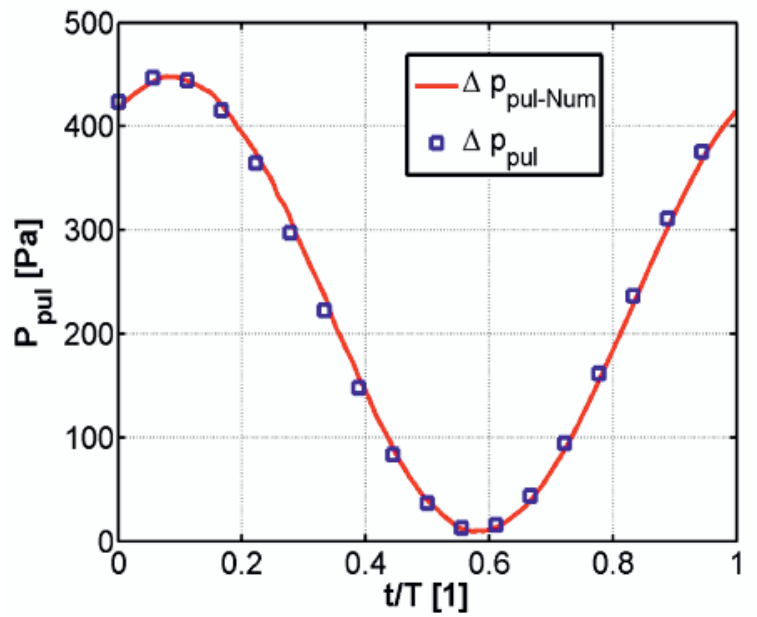

Fig. 11. Comparison of calculated pressure by means of impedance and numerically calculated pressure for regime no.5.

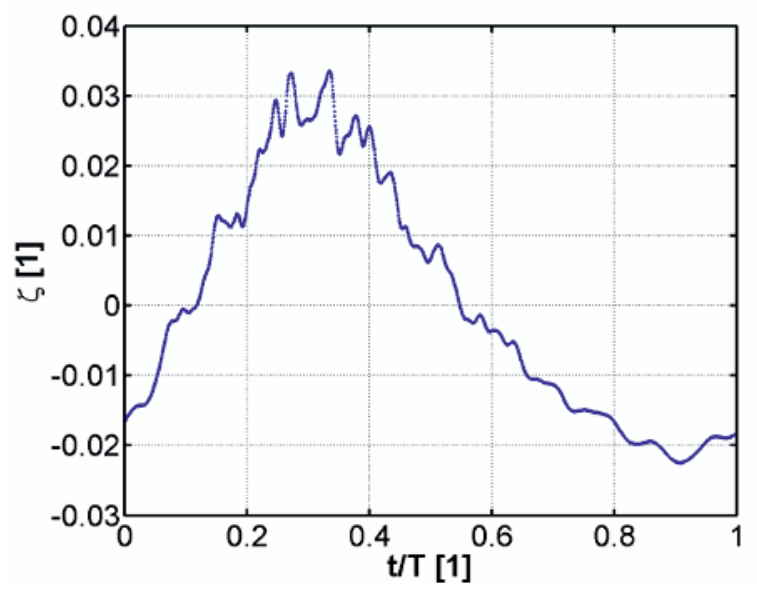

Fig. 12. Course of error for regime no. 5

\section{References}

[1] N. P. Kikas, Solar Energy, 54/No. 4, 209-217 (1995)

[2] D. Haller, P. Woias, N. Kockmann, Inter. J. of Heat and Mass Transfer 52 2678-2689 (2009)

[3] W. Bi, D. Chen, Z. Lin, Inter. J. of hydrog. ener. 1-12 (2009)

[4] S. Maharudraya, S. Jayanti, A. P. Deshpande, J. of Pow. Sour. 138, 1-13 (2004)

[5] M. Olufsen, C. Peskin, W. Kim, E. Pedersen, A. Nadim, J. Larsen, Ann Biomed Eng 28, 1281-99 (2000)

[6] E. Marchandise, M. Willemet, V. Lacroix, Medical Engin. \& Phys. 31, 131-144 (2009)

[7] Y. Huo and G. S. Kassab Am J Physiol Heart Circ Physiol, 292:2623-2633 (2007)

[8] T. Grus, J. Lindner, T. Tosovsky, J. Matecha, V. Rohn, L. Lambert, G. Grusová, Ann. of vascular surgery. 23, 598-605 (2009)

[9] J. Vimmr, A. Jonasova, Math. and comp. in simulation 80, 1324-1336 (2010)

[10] J. M. Lee, M. Y. Kang, H. J. Yang, E. Lee, Med Bio Eng Comput 45, 1071-1078 (2007)

[11] I. E. Idelchik, Handbook of Hydraulic Resistance Begell House (2001) 
[12] D.K. Jamison, J.R. Villemonte, Proc ASCE J Hydraulics Div 97, 1045-1063 (1971)

[13] D.S. Miller, Internal Flow Systems: Design and Performance Prediction Houston, TX: Gulf Publish. (1990)

[14] F. M. White, Fluid Mechanics N. Y. McGraw/Hill. (1994)

[15] CH.J. Morris, F.K. Forster, Exp. in fluids 36, 928937 (2004)

[16] H. Netrebska, Dissertation, CTU in Prague (2011)

[17] J. Novotny, L. Manoch, J. of Mech. Eng. and Autom. 2, 754-761 (2012)

\section{Appendix / used symbols}

$\mathfrak{R}$ - Real part of complex number

$\mathfrak{I}$ - Imaginary part of complex number

Lower index:

AN - Result of analytical solution

NUM - Result of numerical solution (CFD)

pul - Pulsatile

st - Steady component of the pulsatile flow

osc - Oscillating component of the pulsatile flow

Amp - Amplitude

max - Maximum value

$\xi$ - Local loss

$\omega-$ Friction loses and inertia forces of the oscillating component

$\lambda$ - Friction loss

Upper index:

$\wedge$ - Complex number

|| - Straight section of channel

Capital letters - complex variable 